\title{
Validation of serrated polyps (SPs) in Swedish pathology registers
}

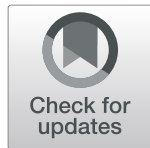

Soran R. Bozorg ${ }^{1 *}$ (D), Mingyang Song 2,3,4 Louise Emilsson $^{1,2,5,6}$ and Jonas F. Ludvigsson 1,7,8,9

\begin{abstract}
Background: Little is known about the natural history of serrated polyps (SPs), partly due to the lack of large-scale epidemiologic data. In this study, we examined the validity of SP identification according to SNOMED (Systematised Nomenclature of Medicine) codes and free text from colorectal histopathology reports.

Methods: Through the ESPRESSO (Epidemiology Strengthened by histoPathology Reports in Sweden) study, we retrieved data on SPs from all pathology departments in Sweden in 2015-2017 by using SNOMED codes and freetext search in colorectal histopathology reports. Randomly selected individuals with a histopathology report of SPs were validated against patient charts using a structured, retrospective review.

Results: SPs were confirmed in 101/106 individuals with a histopathology report of SPs, yielding a positive predictive value (PPV) of $95 \%(95 \% \mathrm{Cl}=89-98 \%)$. By year of diagnosis, the PPV was $89 \%(95 \% \mathrm{Cl}=69-97 \%), 96 \%$ $(95 \% \mathrm{Cl}=81-99 \%)$ and $97 \%(95 \% \mathrm{Cl}=89-99 \%)$ for individuals diagnosed before $2001(n=19)$, between 2001 and $2010(n=26)$ and after $2010(n=61)$, respectively. According to search method, the PPV for individuals identified by SNOMED codes was 100\% $(95 \% \mathrm{Cl}=93-100 \%)$, and $93 \%(95 \% \mathrm{Cl}=86-97 \%)$ using free-text search. Recorded location (colon vs. rectum) was correct in $94 \%$ of all SP histopathology reports $(95 \% \mathrm{Cl}=84-98 \%)$ identified by SNOMED codes. Individuals with SPs were classified into hyperplastic polyps $(n=34 ; 32 \%)$, traditional serrated adenomas $(n=3 ; 3 \%)$, sessile serrated adenomas/polyps (SSA/Ps) $(n=70 ; 66 \%)$, unspecified SPs $(n=3,3 \%)$, and false positive SPS $(n=5,5 \%)$. For individuals identified by SNOMED codes, SSA/Ps were confirmed in 49/52 individuals, resulting in a PPV of $94 \%$ (95\%Cl: $84-98 \%)$. In total, $57 \%$ had $\geq 2$ polyps

$(1: n=44,2-3: n=33$ and $\geq 4: n=27)$. Some $46 \%$ of SPs $(n=71)$ originated from the proximal colon and $24 \%$ were $\geq 10 \mathrm{~mm}$ in size $(n=37)$. Heredity for colorectal cancer, intestinal polyposis syndromes, or both was reported in seven individuals (7\%). Common comorbidities included diverticulosis $(n=45,42 \%)$, colorectal cancer $(n=19,18 \%)$, and inflammatory bowel disease $(n=10,9 \%)$.
\end{abstract}

Conclusion: Colorectal histopathology reports are a reliable data source to identify individuals with SPs.

Keywords: Hyperplastic polyp, Serrated adenoma, Serrated polyp, Sessile serrated adenoma/polyp, Traditional serrated adenoma, Validation

\section{Background}

Colorectal cancer (CRC) is the third most common cancer and the third leading cause of cancer death worldwide. It kills over 600,000 people annually, accounting for $8 \%$ of cancer-related deaths [1]. Adenomatous polyps, now referred to as conventional adenomas, have been regarded as the main precursor of CRC, but in

\footnotetext{
* Correspondence: soranbozorg@gmail.com

'Department of Medical Epidemiology and Biostatistics, Karolinska Institutet, 17177 Solna, Sweden

Full list of author information is available at the end of the article
}

recent years a new pathway to CRC has been identified, termed the serrated pathway $[2,3]$.

Serrated polyps (SPs) are characterised by a saw-toothed appearance of colonic crypts. As per recommendations from the World Health Organisation (WHO) [4], SPs are classified into three subgroups: hyperplastic polyps (HPs), traditional serrated polyps (TSAs) and sessile serrated adenomas/polyps (SSA/Ps)(Fig. 2 in Appendix). The serrated pathway to CRC is mainly believed to originate from SSA/ Ps, which are estimated to represent up to $20 \%$ of all SPs $[3,5]$.

(C) The Author(s). 2019 Open Access This article is distributed under the terms of the Creative Commons Attribution 4.0 International License (http://creativecommons.org/licenses/by/4.0/), which permits unrestricted use, distribution, and 
Some data suggest that cancers evolving through the serrated pathway may account for up to $15-30 \%$ of all CRC cases, and that they are significantly overrepresented in interval cancers [6], i.e. CRC occurring before the next recommended screening after an initially negative finding. Even though the adenoma-carcinoma pathway still accounts for the majority of the CRC burden, a recent study comparing the risk of $\mathrm{CRC}$ development found that the increased risk of CRC in individuals with SPs is similar or higher than that seen in individuals with conventional adenomas [7].

Little is known about the natural history of SP, which may in part be due to the lack of availability of largescale data. Through the ESPRESSO (Epidemiology Strengthened by histoPathology) study [8], we contacted all pathology departments $(n=28)$ in Sweden to construct a cohort of individuals with an SP diagnosis according to computerised histopathology reports. We then retrieved patient charts from 106 randomly selected individuals with a record of SP. The primary purpose of this study was to validate SP diagnosis according to computerised histopathology reports against patient chart data. A secondary aim was to describe the characteristics of individuals with SPs.

\section{Methods}

We validated SP diagnosis based on computerised histopathology reports in a random subset of individuals through a structured, retrospective review of histopathology reports and patient charts.

\section{Study population}

The ESPRESSO study consists of gastrointestinal histopathology reports from 2.2 million unique individuals with a total of 6.1 million separate data entries. Some $53.9 \%$ of individuals had been biopsied more than once. Data on gastrointestinal histopathology reports were collected between October 12, 2015 and April 15, 2017 from all pathology departments in Sweden $(n=28)$. Overall we had data on 1,618,953 colon biopsies and 771,511 rectal biopsies [8]. Through the unique personal identity number [9] assigned to all Swedish residents, histopathology data were linked to the Swedish national health registers (Patient Register [10], Cause of Death Register [11], Cancer Register [12], Medical Birth Register [13], Prescribed Drug Register [14], The LISA database with socioeconomic data [15], as well as the Total Population Register [16]). Details about ESPRESSO and registry linkage have been described previously [8].

For the current study on SPs, we included individuals with a colorectal biopsy (topography codes: T67-68) with the following Systematised Nomenclature of Medicine (SNOMED) codes: M82160, M8216, M82130, M8213. We also included individuals with a colorectal biopsy of which the histopathology report free text listed "serrated polyp" (Swedish "sågtand(ad)").

\section{Study sample}

Power calculation using EpiTools [17] indicated a minimum of 139 individuals were needed to obtain a positive predictive value (PPV) for SP of $90 \%$ with a $95 \%$ confidence interval $(95 \% \mathrm{CI})$ range of $85-95 \%$ (using an alpha of 0.05 and a beta of 0.20 ). For this validation, we requested patient charts from a random sample of 160 individuals with a histopathology report of SPs from five Swedish counties. We were able to retrieve patient chart data from 126 individuals, out of which 106 had sufficient information for our validation (Fig. 1).

\section{Case definition}

We defined a true SP as having a consistent histopathology report and a patient chart supporting an SP diagnosis. Individuals with an SP diagnosis could have one or multiple SPs. Assessment of histopathology reports and patient charts was executed by the principal author (SRB). Uncertain cases were discussed with JFL and MS. If no consensus was reached, the case was considered inconsistent with SP.

\section{Data elements}

Data from patient charts were extracted using a standardised form, similar to the form used by Svensson et al. in their validation of microscopic colitis [18]. The starting point of data extraction was set to 2 years before the diagnosis until March 2018. The data from the patient charts mainly included patient history, laboratory data, referral letters and endoscopy and histopathology reports. Individuals were excluded in the absence of a histopathology report or insufficient/incomplete data.

\section{Statistics}

The main outcome of this study was the PPV for SP diagnosis in the 106 individuals with patient charts containing sufficient data. To identify any potential differences, results were stratified according to search method (SNOMED codes or free-text search). Given the changing nomenclature of SPs over time, we also analysed the data by year of diagnosis. For individuals identified by SNOMED codes, we validated the SP location by comparing the topography code with the patient chart. SNOMED codes were also used to identify SSA/Ps, for which a separate PPV was calculated. We estimated 95\%CIs with the Wilson score interval [19] using EpiTools [20].

In addition to retrieving colonoscopy and histopathology reports, we collected data on sex, age, year of diagnosis, smoking, obesity, comorbidity, diagnostic tools and indication for endoscopy. For evaluation of anaemia, 


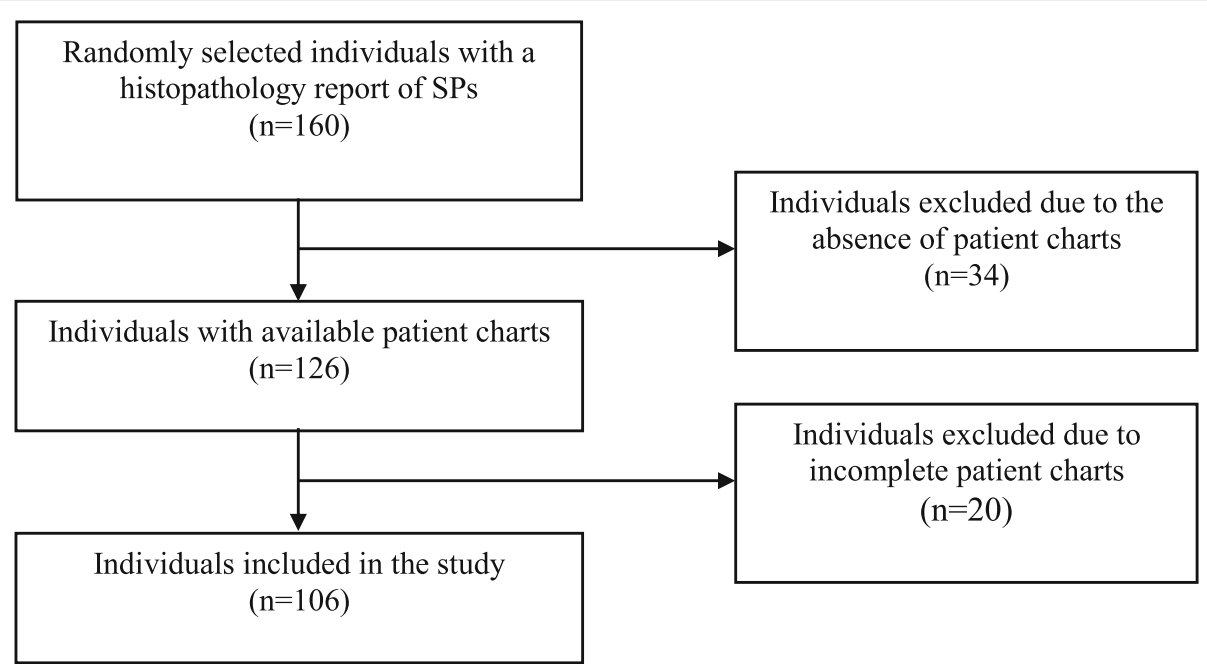

Fig. 1 Flowchart for inclusion of individuals in the study. (Abbreviations: SPs = Serrated polyps)

we used $132 \mathrm{~g} / \mathrm{L}$ for men and $122 \mathrm{~g} / \mathrm{L}$ for women as the lower limits of normal haemoglobin concentration as proposed by Beutler and Waalen [21]. The size of polyp characteristics was determined as either larger or smaller than $10 \mathrm{~mm}$ as this size has been proposed as the threshold for determining the future management of SPs [22]. Other aspects investigated were number of polyps $(0,1,2-3$ or $\geq 4)$, location (proximal, distal, rectal) and grade of dysplasia (none, low, high). The proximal colon was defined as the ileocecal valve until the splenic flexure, followed by the distal colon until the last $10 \mathrm{~cm}$ of the gastrointestinal tract that represent the rectum.

For the descriptive analysis, we calculated the population and polyp characteristics according to SP subgroups. To reflect the previous version of the WHO recommendations on SP classification, SPs described as serrated adenomas (SAs) or mixed polyps with a serrated component were deemed consistent with SSA/P [23]. Nonetheless, data were also analysed separately for these polyp subgroups. Data on false positive SPs were also presented separately.

\section{Results}

Data

The charts of 106 individuals were retrieved from pathology centres distributed in five counties in Sweden: Dalarna, Norrbotten, Skaraborg, Stockholm and Örebro.

\section{Positive predictive value (PPV)}

SPs were confirmed in 101/106 individuals, yielding a PPV of 95\% (95\%CI =89-98\%) (Table 1). Of the five individuals with false positive SPs, one had SP ruled out by the pathologist. The other four individuals had SPs mentioned in the histopathology report but sufficient evidence to confirm the diagnosis was lacking. No false positive case was found among individuals identified by SNOMED codes $(n=52)$, resulting in a PPV of $100 \%$ $(95 \% \mathrm{CI}=93-100 \%)$. For individuals identified by freetext search of histopathology reports $(n=76)$, the PPV was $93 \%(95 \% C I=86-97)$. Out of these, 22 individuals also had a SNOMED code.

By year of diagnosis, the PPV was $89 \%(95 \% \mathrm{CI}=$ $69-97 \%), 96 \%(95 \% \mathrm{CI}=81-99 \%)$ and $97 \%(95 \% \mathrm{CI}=$ 89-99\%) for individuals diagnosed before $2001 \quad(n=$ 19), between 2001 and $2010(n=26)$ and after 2010 $(n=61)$, respectively. All individuals diagnosed before 2000 were identified by free-text entries alone, while $69 \% \quad(n=36)$ of the individuals identified by SNOMED codes were diagnosed after 2010. For individuals identified by SNOMED codes, the recorded location was accurate in 49/52 (94\%, 95\%CI $=84-$ 98\%) cases of all SP histopathology reports. The three individuals with an incorrect recorded topography code had been biopsied in the distal (sigmoid) colon but recorded as having their polyp in the rectum (T68). Only five individuals had a subsitespecific topography code within the colon (T671T677), all of which were accurate.

Most individuals with SSA/Ps were identified by SNOMED codes $(n=49,70 \%)$, whereas most individuals with HPs were identified by free-text searches ( $n$ $=31,91 \%)$. Of all individuals identified by SNOMED codes, SSA/Ps were confirmed in 49/52 individuals, resulting in a PPV of $94 \%(95 \% \mathrm{CI}=84-98 \%)$. The false positive cases consisted of two TSAs $(n=2)$ and one $\operatorname{HP}(n=1)$. 
Table 1 Positive predictive value of serrated polyps in Swedish histopathology reports

\begin{tabular}{|c|c|c|c|}
\hline Variable & SNOMED codes M8213(0)/M8216(0) & Free text "serrated" & Total \\
\hline Individuals & $52(49 \%)$ & $76(72 \%)$ & $106(100 \%)$ \\
\hline \multicolumn{4}{|l|}{ By subtype } \\
\hline Hyperplastic polyp & $5(10 \%)$ & $31(41 \%)$ & $34(32 \%)$ \\
\hline Traditional serrated adenoma & $2(4 \%)$ & $3(4 \%)$ & $3(3 \%)$ \\
\hline Sessile serrated adenoma/polyp & $49(94 \%)$ & $40(53 \%)$ & $70(66 \%)$ \\
\hline Unspecified serrated polyp & $0(0 \%)$ & $3(4 \%)$ & $3(3 \%)$ \\
\hline False positive serrated polyp & $0(0 \%)$ & $5(7 \%)$ & $5(5 \%)$ \\
\hline PPV, SP diagnosis & $52 / 52,100 \%(95 \% \mathrm{Cl}=93-100 \%)$ & $71 / 76,93 \%(95 \% \mathrm{Cl}=86-97 \%)$ & $101 / 106,95 \%(95 \% \mathrm{Cl}=89-98 \%)$ \\
\hline \multicolumn{4}{|l|}{ By year of diagnosis } \\
\hline$<2000$ & $0 / 0$ & $17 / 19,89 \%$ & $17 / 19,89 \%$ \\
\hline $2000-2009$ & $16 / 16,100 \%$ & $10 / 11,91 \%$ & $25 / 26,96 \%$ \\
\hline$\geq 2010$ & $36 / 36,100 \%$ & $44 / 46,96 \%$ & $59 / 61,97 \%$ \\
\hline PPV, location & $49 / 52,94 \%(95 \% \mathrm{Cl}=84-98 \%)$ & - & - \\
\hline \multicolumn{4}{|l|}{ By topography code } \\
\hline T67 & $25 / 25,100 \%$ & - & - \\
\hline T671 & $1 / 1,100 \%$ & - & - \\
\hline T672 & $3 / 3,100 \%$ & - & - \\
\hline T677 & $2 / 2,100 \%$ & - & - \\
\hline T68 & $18 / 21,86 \%$ & - & - \\
\hline PPV, SSA/P diagnosis & $49 / 52,94 \%(95 \% \mathrm{Cl}=84-98 \%)$ & - & - \\
\hline
\end{tabular}

Abbreviations: $\mathrm{Cl}=$ Confidence interval; PPV = Positive predictive value; SP = Serrated polyp; SNOMED = Systematised Nomenclature of Medicine

Table 2 Demographics of individuals with serrated polyps according to Swedish histopathology reports

\begin{tabular}{|c|c|c|c|c|c|c|c|c|c|}
\hline \multirow[t]{2}{*}{ Variable } & \multirow[t]{2}{*}{$\mathrm{HP}$} & \multirow[t]{2}{*}{ TSA } & \multicolumn{4}{|l|}{ SSA/P } & \multirow[t]{2}{*}{$U$} & \multirow[t]{2}{*}{$\mathrm{F}$} & \multirow[t]{2}{*}{ Total } \\
\hline & & & SSA/P & SA & Mixed & Total & & & \\
\hline INDIVIDUALS & $34(32 \%)$ & $3(3 \%)$ & $8(8 \%)$ & $51(48 \%)$ & $12(11 \%)$ & $70(66 \%)$ & $3(3 \%)$ & $5(5 \%)$ & $106(100 \%)$ \\
\hline \multicolumn{10}{|l|}{ Year of diagnosis } \\
\hline Min & 1989 & 2015 & 2011 & 2002 & 2008 & 2002 & 2005 & 1991 & 1989 \\
\hline Median & 2003 & 2015 & 2014 & 2013 & 2014 & 2014 & 2008 & 2007 & 2012 \\
\hline Max & 2016 & 2016 & 2016 & 2015 & 2015 & 2016 & 2014 & 2014 & 2016 \\
\hline \multicolumn{10}{|l|}{ Age } \\
\hline Min & 39 & 52 & 59 & 41 & 53 & 41 & 62 & 35 & 35 \\
\hline Median & 68 & 58 & 64 & 74 & 74 & 74 & 65 & 73 & 70 \\
\hline Max & 82 & 66 & 78 & 93 & 85 & 93 & 70 & 87 & 93 \\
\hline \multicolumn{10}{|l|}{ Sex } \\
\hline Female & $15(44 \%)$ & $0(0 \%)$ & $5(63 \%)$ & $26(51 \%)$ & $5(42 \%)$ & 35 (50\%) & $3(100 \%)$ & $1(20 \%)$ & $50(47 \%)$ \\
\hline \multicolumn{10}{|l|}{ Detection procedure } \\
\hline Colonoscopy & $28(82 \%)$ & $2(67 \%)$ & 7 (88\%) & $40(78 \%)$ & $10(83 \%)$ & $57(87 \%)$ & $3(100 \%)$ & $5(100 \%)$ & $86(81 \%)$ \\
\hline Partial lower endoscopy* & $5(15 \%)$ & $1(33 \%)$ & $0(0 \%)$ & $9(18 \%)$ & $0(0 \%)$ & $9(0 \%)$ & $0(0 \%)$ & $0(0 \%)$ & $15(14 \%)$ \\
\hline Colectomy & $1(3 \%)$ & $0(0 \%)$ & $1(13 \%)$ & $2(4 \%)$ & $2(17 \%)$ & $4(13 \%)$ & $0(0 \%)$ & $0(0 \%)$ & $5(5 \%)$ \\
\hline
\end{tabular}

Abbreviations: $\mathrm{F}=$ False positive serrated polyp; HP = Hyperplastic polyp; SA = Serrated adenoma; SSA/P = Sessile serrated adenoma/polyp; TSA = Traditional serrated adenoma; $\mathrm{U}=$ Unspecified serrated polyp. *Sigmoidoscopy, rectoscopy or proctoscopy 


\section{Demographics and risk factors}

Of the 106 validated individuals, 50 were female (47\%) and the median age at diagnosis was 70 years (Table 2). Most SP cases were diagnosed through colonoscopy $(n=86,81 \%)$, with smaller proportions diagnosed through partial lower endoscopy (sigmoidoscopy, rectoscopy or proctoscopy, $n=15,14 \%$ ) or hemicolectomy $(n=5,5 \%)$. The data were stratified as follows: HP $(n=$ $34,32 \%)$, TSA $(n=3,3 \%), \mathrm{SSA} / \mathrm{P}(n=70,66 \%)$, unspecified SP $(n=3,3 \%)$, and false positive SP $(n=5,5 \%)$. The SSA/P subgroup also included polyps described as serrated adenomas $(n=51)$ and mixed polyps $(n=12)$. Because some individuals had polyps of different subtypes $(n=9)$, the sum of individuals in the subgroups exceeds the total number of individuals reviewed. Notably, the HP subgroup was diagnosed earlier than the SPs overall (median year: 2003 vs. 2012) and there were no polyps specifically described as an SSA/P or TSA before 2011. Polyps described specifically as serrated adenomas were reported as early as 2002. Otherwise, population characteristics were similar in the different SP subgroups.

At diagnosis, 16 (15\%) individuals were current smokers, whereas 14 (13\%) had a record of earlier smoking (Table 3). Obesity (body mass index, BMI $\geq 30$ or indication of obesity in the patient chart) was seen in 12 individuals (11\%). Heredity for CRC, intestinal polyposis syndromes, or both was reported in seven individuals (7\%). Common comorbidities consisted of diverticulosis $(n=45,42 \%)$, conventional adenomas $(n=33,31 \%), \quad$ CRC $\quad(n=19,18 \%)$ and inflammatory bowel disease (IBD) $(n=10,9 \%)$. Comorbidities were defined as having a diagnosis prior to or in conjunction with a diagnosis of SP, except for conventional adenomas for which prior diagnoses were not considered.

\section{Indications and symptoms}

Most individuals underwent endoscopy for clinical symptoms $(n=64,60 \%)$ (Table 4). For individuals with symptomatic indications and SP as their only significant endoscopic finding $(n=28)$, the most frequent symptoms were change in stool form (diarrhoea or obstipation, $n=$ $21,75 \%$ ), change in stool colour (haematochezia or melena, $n=14,50 \%)$ and anaemia $(n=11,39 \%)$. Endoscopies carried out due to an asymptomatic indication $(n=$ $39,37 \%)$ mostly consisted of surveillance endoscopies due to a history of previous polyps or adenomas $(n=18,46 \%)$, a history of CRC $(n=3,8 \%)$ or a history of IBD $(n=5$, $13 \%)$. CRC screening was also a frequent indication of asymptomatic endoscopies $(n=8,21 \%)$, out of which six individuals also had a positive faecal occult blood test (FOBT) prior to the endoscopy.

The most frequent symptoms in individuals in our cohort, regardless of endoscopy indication, were change in stool form $(n=47,44 \%)$, change in stool colour $(n=36$, $34 \%)$, anaemia $(n=30,28 \%)$, abdominal pain $(n=22$, $21 \%)$, weight loss $(n=9,8 \%)$ and fatigue $(n=8,8 \%)$. Other less frequent symptoms included nausea $(n=4$, $4 \%)$, anal burning $(\mathrm{n}=2,2 \%)$, fever $(n=1,1 \%)$, loss of appetite $(\mathrm{n}=1,1 \%)$ and dyspnoea $(\mathrm{n}=1,1 \%)$. Fifteen individuals (14\%) had a positive FOBT before endoscopy. In total, clinical signs of gastrointestinal bleeding (change in stool colour, anaemia or FOBT) were seen in 58 (55\%) individuals in total and in 28 (61\%) individuals with SP as their only endoscopic finding.

Table 3 Risk factors and comorbidity of individuals with serrated polyps according to Swedish histopathology reports

\begin{tabular}{|c|c|c|c|c|c|c|c|c|c|}
\hline \multirow[t]{2}{*}{ Variable } & \multirow[t]{2}{*}{$\mathrm{HP}$} & \multirow[t]{2}{*}{ TSA } & \multicolumn{4}{|l|}{ SSA/P } & \multirow[t]{2}{*}{ U } & \multirow[t]{2}{*}{$\mathrm{F}$} & \multirow[t]{2}{*}{ Total } \\
\hline & & & SSA/P & SA & Mixed & Total & & & \\
\hline INDIVIDUALS & $34(32 \%)$ & $3(3 \%)$ & $8(8 \%)$ & $51(48 \%)$ & 12 (11\%) & $70(66 \%)$ & $3(3 \%)$ & $5(5 \%)$ & $106(100 \%)$ \\
\hline \multicolumn{10}{|l|}{ Smoking } \\
\hline Current & $8(24 \%)$ & $1(33 \%)$ & $1(13 \%)$ & $2(4 \%)$ & $4(33 \%)$ & $7(10 \%)$ & $2(67 \%)$ & $0(0 \%)$ & $16(15 \%)$ \\
\hline Previous & $5(15 \%)$ & $2(67 \%)$ & $3(38 \%)$ & $5(10 \%)$ & $2(17 \%)$ & $10(14 \%)$ & $0(0 \%)$ & $0(0 \%)$ & $14(13 \%)$ \\
\hline \multicolumn{10}{|l|}{ BMI } \\
\hline Obese $e^{*}$ & $3(9 \%)$ & $2(67 \%)$ & $2(25 \%)$ & $5(10 \%)$ & $1(8 \%)$ & $8(11 \%)$ & 1 (33\%) & $0(0 \%)$ & 12 (11\%) \\
\hline \multicolumn{10}{|l|}{ Heredity } \\
\hline$C R C+{ }^{\S}$ & $2(6 \%)$ & $0(0 \%)$ & $2(25 \%)$ & $1(2 \%)$ & $1(8 \%)$ & $4(6 \%)$ & $1(33 \%)$ & $0(0 \%)$ & $7(7 \%)$ \\
\hline \multicolumn{10}{|l|}{ Comorbidity } \\
\hline Diverticulosis & $9(26 \%)$ & $1(33 \%)$ & $4(50 \%)$ & $24(47 \%)$ & $8(67 \%)$ & 36 (51\%) & $1(33 \%)$ & $1(20 \%)$ & $45(42 \%)$ \\
\hline$C R C$ & $3(9 \%)$ & $1(33 \%)$ & $3(38 \%)$ & $10(20 \%)$ & $3(25 \%)$ & 15 (21\%) & 2 (67\%) & $0(0 \%$ & 19 (18\%) \\
\hline IBD & $4(12 \%)$ & $0(0 \%)$ & $0(0 \%)$ & $5(10 \%)$ & $1(8 \%)$ & $6(9 \%)$ & $0(0 \%)$ & $0(0 \%)$ & $10(9 \%)$ \\
\hline
\end{tabular}

Abbreviations: $\mathrm{BMI}=$ Body mass index; $\mathrm{CRC}=$ Colorectal cancer; $\mathrm{F}=$ False positive serrated polyp; $\mathrm{HP}=$ Hyperplastic polyp; IBD = Inflammatory bowel disease; $\mathrm{SA}=$ Serrated adenoma; SSA/P = Sessile serrated adenoma/polyp; TSA = Traditional serrated adenoma; $\mathrm{U}=$ Unspecified serrated polyp. ${ }^{*} B M I \geq 30$ or indication of obesity in patient chart, ${ }^{\S} \mathrm{CRC}$ and/or intestinal polyposis syndromes 
Table 4 Endoscopy indication and symptoms of individuals with serrated polyps according to Swedish histopathology reports

\begin{tabular}{|c|c|c|c|c|c|c|c|c|c|}
\hline \multirow[t]{2}{*}{ Variable } & \multirow[t]{2}{*}{$\mathrm{HP}$} & \multirow[t]{2}{*}{ TSA } & \multicolumn{4}{|l|}{ SSA/P } & \multirow[t]{2}{*}{ U } & \multirow[t]{2}{*}{$\mathrm{F}$} & \multirow[t]{2}{*}{ Total } \\
\hline & & & SSA/P & SA & Mixed & Total & & & \\
\hline INDIVIDUALS & $34(32 \%)$ & $3(3 \%)$ & $8(8 \%)$ & $51(48 \%)$ & $12(11 \%)$ & $70(66 \%)$ & $3(3 \%)$ & $5(5 \%)$ & $106(100 \%)$ \\
\hline \multicolumn{10}{|l|}{ Indication } \\
\hline Symptomatic & 18 (53\%) & $1(33 \%)$ & $3(38 \%)$ & 35 (69\%) & $8(67 \%)$ & 45 (64\%) & $1(33 \%)$ & $4(80 \%)$ & $64(60 \%)$ \\
\hline Non-symptomatic* & 15 (44\%) & $2(67 \%)$ & $5(63 \%)$ & $14(27 \%)$ & $4(33 \%)$ & $23(33 \%)$ & $2(67 \%)$ & $1(20 \%)$ & 39 (37\%) \\
\hline Unspecified & $1(3 \%)$ & $0(0 \%)$ & $0(0 \%)$ & $2(4 \%)$ & $0(0 \%)$ & $2(3 \%)$ & $0(0 \%)$ & $0(0 \%)$ & $3(3 \%)$ \\
\hline \multicolumn{10}{|l|}{ Symptoms } \\
\hline Change in stool form & $16(47 \%)$ & $1(33 \%)$ & $2(25 \%)$ & $23(45 \%)$ & $5(42 \%)$ & $30(43 \%)$ & $0(0 \%)$ & $2(40 \%)$ & 47 (44\%) \\
\hline Change in stool colour & $9(26 \%)$ & $0(0 \%)$ & $3(38 \%)$ & $22(43 \%)$ & $3(25 \%)$ & 27 (39\%) & $1(33 \%)$ & $1(20 \%)$ & $36(34 \%)$ \\
\hline Abdominal pain & $5(15 \%)$ & $0(0 \%)$ & $4(50 \%)$ & $10(20 \%)$ & $2(17 \%)$ & 15 (21\%) & $1(33 \%)$ & $1(20 \%)$ & $22(21 \%)$ \\
\hline Weight loss & $2(6 \%)$ & $0(0 \%)$ & $0(0 \%)$ & $6(12 \%)$ & $1(8 \%)$ & $7(10 \%)$ & $1(33 \%)$ & $0(0 \%)$ & $9(8 \%)$ \\
\hline Fatigue & $2(6 \%)$ & $0(0 \%)$ & $0(0 \%)$ & $4(8 \%)$ & $2(17 \%)$ & $6(9 \%)$ & $0(0 \%)$ & $0(0 \%)$ & $8(8 \%)$ \\
\hline Anaemia & $6(18 \%)$ & $0(0 \%)$ & 1 (13\%) & 18 (35\%) & $4(33 \%)$ & 22 (31\%) & $1(33 \%)$ & $2(40 \%)$ & $30(28 \%)$ \\
\hline Positive FOBT & $4(12 \%)$ & $3(100 \%)$ & 1 (13\%) & $4(8 \%)$ & $3(25 \%)$ & $8(11 \%)$ & $2(67 \%)$ & $0(0 \%)$ & 15 (14\%) \\
\hline Others $^{\S}$ & $4(12 \%)$ & $0(0 \%)$ & $0(0 \%)$ & $5(10 \%)$ & $0(0 \%)$ & $5(7 \%)$ & $0(0 \%)$ & $0(0 \%)$ & $8(8 \%)$ \\
\hline
\end{tabular}

Abbreviations: $\mathrm{F}=$ False positive serrated polyp; FOBT = Faecal occult blood test; $\mathrm{HP}=$ Hyperplastic polyp; SA = Serrated adenoma; SSA $/ \mathrm{P}=$ Sessile serrated adenoma/polyp; TSA = Traditional serrated adenoma; $\mathrm{U}=$ Unspecified serrated polyp. ${ }^{*}$ Polyp surveillance, $C R C$ screening, etc., ${ }^{S}$ Fever, burning, nausea, etc.

\section{Polyp characteristics}

According to endoscopy reports, 44 (42\%) individuals had one polyp at diagnosis, 33 (31\%) had 2-3 polyps and $27(25 \%)$ had $\geq 4$ polyps (Table 5$)$. Two persons with a false positive SP had no certain polyps. The total number of polyps was 155 , which could be classified into HPs $(n=61,39 \%)$, TSAs $(n=3,2 \%)$, SSA/Ps $(n=80,52 \%)$, unspecified SPs $(n=8,5 \%)$ and false positive SPs $(n=3,2 \%)$. The size of the polyps was determined as either large $(\geq 10 \mathrm{~mm})$ or small $(<10$ $\mathrm{mm})$. In all, there were 58 (37\%) small polyps and 37 (24\%) large polyps. Only four (7\%) HPs were considered large, out of which two were proximal. In contrast, all TSAs were large $(\mathrm{n}=3,100 \%)$, whereas SSA/ Ps presented a more even distribution regarding size (small: $n=31,39 \%$, large: $n=28,35 \%$ ). In terms of location unspecified SPs were predominantly found in the proximal colon $(n=7,88 \%)$, whereas SSA/Ps were generally found either proximally $(n=39,49 \%)$ or rectally $(n=26,33 \%)$. TSAs were seen in the distal colon $(n=1,33 \%)$ or rectum $(n=2,67 \%)$, whereas HPs were relatively evenly distributed.

Evaluating the grade of dysplasia, 26 polyps had no sign of dysplasia (17\%). Low-grade dysplasia was seen in $70(45 \%)$ polyps and high grade in $5(3 \%)$. Polyps with no dysplasia were overrepresented among HPs $(n=16$, $26 \%)$ and unspecified SPs $(n=5,63 \%)$. In $43(70 \%)$ HPs degree of dysplasia was not specified. The number of polyps with unspecified degree of dysplasia in the other subgroups was $0(0 \%)$ for TSA, 9 (11\%) for SSA/P and 2 (25\%) for unspecified SP. Most TSAs and SSA/Ps exhibited low-grade dysplasia (TSA: $n=3,100 \%$; SSA/P: $n=$
$61,76 \%)$; cases of high-grade dysplasia were only seen in SSA/Ps $(n=5,6 \%)$.

\section{Discussion}

Our study found a high PPV (95\%, 95\%CI: 89-98\%) for SPs according to colorectal histopathology reports based on SNOMED codes and free-text searches. The high PPV was similar over time. This finding suggests that histopathology reports are a reliable source to identify individuals with SPs. The PPV of this study is comparable with that of other gastrointestinal diagnoses based on histopathology: celiac disease (PPV 95\%) and microscopic colitis (PPV 95\%) [18, 24]. The high specificity for SPs is not surprising given that the assignment of the SNOMED code and free-text diagnosis is already based on histopathological evaluation.

As to search method, the use of SNOMED codes to identify individuals with SPs had a higher specificity than the use of free-text search (PPV: $100 \%$ vs. 93\%), but still the PPV using free text is consistent with the accuracy of having a physician-assigned diagnosis in the Swedish Patient Register (95\%CI PPV $=85-95 \%)$ [10]. Furthermore, the high PPV of SSA/P among individuals identified through SNOMED codes (94\%, 95\%CI: 84-98\%) indicates that an exclusive use of SNOMED codes can serve to target these polyps specifically. For individuals identified by SNOMED codes, the corresponding topography codes can also be used to determine the location of the SPs and SSA/Ps (PPV: 94\%; 95\%CI =84-98\%). The cases of incorrect topography codes exclusively concerned individuals with a rectal topography code (T68), which were classified as distal (sigmoidal) according to 
Table 5 Characteristics of serrated polyps

\begin{tabular}{|c|c|c|c|c|c|c|c|c|c|}
\hline \multirow[t]{2}{*}{ Variable } & \multirow[t]{2}{*}{ HP } & \multirow[t]{2}{*}{ TSA } & \multicolumn{4}{|l|}{ SSA/P } & \multirow[t]{2}{*}{$U$} & \multirow[t]{2}{*}{$\mathrm{F}$} & \multirow[t]{2}{*}{ Total } \\
\hline & & & SSA/P & SA & Mixed & Total & & & \\
\hline Individuals & $34(32 \%)$ & $3(3 \%)$ & $8(3 \%)$ & $51(48 \%)$ & $12(11 \%)$ & $70(66 \%)$ & $3(3 \%)$ & $5(5 \%)$ & $106(100 \%)$ \\
\hline \multicolumn{10}{|l|}{ Number of polyps* } \\
\hline 0 & $0(0 \%)$ & $0(0 \%)$ & $0(0 \%)$ & $0(0 \%)$ & $0(0 \%)$ & $0(0 \%)$ & $0(0 \%)$ & $2(40 \%)$ & $2(2 \%)$ \\
\hline 1 & $8(24 \%)$ & $2(67 \%)$ & $4(50 \%)$ & $24(47 \%)$ & $5(42 \%)$ & $33(47 \%)$ & $0(0 \%)$ & $1(20 \%)$ & $44(42 \%)$ \\
\hline $2-3$ & $11(32 \%)$ & $1(33 \%)$ & $2(25 \%)$ & $15(29 \%)$ & $4(33 \%)$ & $21(30 \%)$ & $1(33 \%)$ & $2(40 \%)$ & $33(31 \%)$ \\
\hline$\geq 4$ & $15(44 \%)$ & $0(0 \%)$ & $2(25 \%)$ & $12(24 \%)$ & $3(25 \%)$ & $16(23 \%)$ & $2(67 \%)$ & $0(0 \%)$ & $27(25 \%)$ \\
\hline \multicolumn{10}{|l|}{ Simultaneous findings } \\
\hline Conventional adenoma & $15(44 \%)$ & $0(0 \%)$ & $0(0 \%)$ & $17(33 \%)$ & $5(42 \%)$ & $22(31 \%)$ & $0(0 \%)$ & $1(20 \%)$ & $33(31 \%)$ \\
\hline$C R C$ & $1(3 \%)$ & $0(0 \%)$ & $3(38 \%)$ & $5(10 \%)$ & $1(8 \%)$ & $8(11 \%)$ & $1(33 \%)$ & $0(0 \%)$ & $10(9 \%)$ \\
\hline POLYPS & 61 (39\%) & $3(2 \%)$ & $11(7 \%)$ & $56(36 \%)$ & $13(8 \%)$ & 80 (52\%) & $8(5 \%)$ & $3(2 \%)$ & $155(100 \%)$ \\
\hline \multicolumn{10}{|l|}{ Size } \\
\hline$<10$ & 26 (43\%) & $0(0 \%)$ & $5(45 \%)$ & $24(43 \%)$ & $2(15 \%)$ & 31 (39\%) & $0(0 \%)$ & $1(33 \%)$ & $58(37 \%)$ \\
\hline$\geq 10$ & $4(7 \%)$ & $3(100 \%)$ & $5(45 \%)$ & 16 (29\%) & 7 (54\%) & 28 (35\%) & $2(25 \%)$ & $0(0 \%)$ & 37 (24\%) \\
\hline Unspecified & 31 (51\%) & $0(0 \%)$ & $1(9 \%)$ & 16 (29\%) & $4(31 \%)$ & $21(26 \%)$ & $6(75 \%)$ & $2(67 \%)$ & 60 (39\%) \\
\hline \multicolumn{10}{|l|}{ Location } \\
\hline Proximal & 25 (41\%) & $0(0 \%)$ & $9(82 \%)$ & 23 (41\%) & 7 (54\%) & 39 (49\%) & 7 (88\%) & $0(0 \%)$ & 71 (46\%) \\
\hline Distal & 25 (41\%) & $1(33 \%)$ & $2(18 \%)$ & $8(14 \%)$ & $5(38 \%)$ & 15 (19\%) & $1(13 \%)$ & $2(67 \%)$ & 44 (28\%) \\
\hline Rectal & 11 (18\%) & $2(67 \%)$ & $0(0 \%)$ & $25(45 \%)$ & $1(8 \%)$ & 26 (33\%) & $0(\%)$ & 1 (33\%) & 40 (26\%) \\
\hline Unspecified & $0(0 \%)$ & $0(0 \%)$ & $0(0 \%)$ & $0(0 \%)$ & $0(0 \%)$ & $0(0 \%)$ & $0(0 \%)$ & $0(0 \%)$ & $0(0 \%)$ \\
\hline \multicolumn{10}{|l|}{ Dysplasia } \\
\hline None & $16(26 \%)$ & $0(0 \%)$ & $2(18 \%)$ & $3(5 \%)$ & $0(0 \%)$ & $5(6 \%)$ & $5(63 \%)$ & $0(0 \%)$ & $26(17 \%)$ \\
\hline Low & $2(3 \%)$ & 3 (100\%) & $4(36 \%)$ & 45 (80\%) & 12 (92\%) & 61 (76\%) & $1(13 \%)$ & $3(100 \%)$ & 70 (45\%) \\
\hline High & $0(0 \%)$ & $0(0 \%)$ & $1(9 \%)$ & $3(5 \%)$ & $1(8 \%)$ & $5(6 \%)$ & $0(0 \%)$ & $0(0 \%)$ & $5(3 \%)$ \\
\hline Unspecified & 43 (70\%) & $0(0 \%)$ & $4(36 \%)$ & $5(9 \%)$ & $0(0 \%)$ & $9(11 \%)$ & $2(25 \%)$ & $0(0 \%)$ & 54 (35\%) \\
\hline
\end{tabular}

Abbreviations: $\mathrm{CRC}=$ Colorectal cancer; $\mathrm{F}=$ False positive serrated polyp; HP = Hyperplastic polyp; SA = Serrated adenoma; SSA/P = Sessile serrated adenoma/polyp; TSA = Traditional serrated adenoma; $\mathrm{U}=$ Unspecified serrated polyp. *Includes conventional adenomas

our validation. This discrepancy occurred because we mainly used endoscopy reports to determine the macroscopic location of the polyps, whereas topography codes are assigned by the pathologist and sometimes based on histological appearance.

Subsequent to the recognition of the different SP subgroups, several studies have investigated their respective prevalence. HPs have consistently been shown to be the most common subtype, representing $70-90 \%$ of all SPs [25-27]. Likewise, SSA/Ps have been shown to represent up to $10-25 \%$ of all SPs while TSAs represent about $1 \%$ [25-29]. In our study, we primarily targeted SSA/Ps. As such, we did not include SNOMED codes for HPs. Consequently, the proportion of HPs in our cohort does not reflect the overall proportion among SPs, as HPs are likely to have been included when they have been described as "serrated" in the histopathology report. As a result, most individuals with HPs have been identified by free-text searches $(n=31,91 \%)$.
Given the evolving nomenclature of SPs, a large number of polyps in our study were described following the previous version of the $\mathrm{WHO}$ classification of colorectal polyps published in 2000 [23]. This version recognised HPs separately and SAs as a subtype under adenomas. Within the SA subtype, there was no differentiation between SSA/Ps and TSAs. As such, polyps described as serrated adenomas can represent any of these two. However, given the predominate prevalence of SSA/Ps, it is reasonable to assume that the number of TSAs described as serrated adenomas is small. It is also reassuring to note that the specific SP descriptions correlated well with the publication year of the different WHO classifications, i.e. polyps described as serrated adenomas began to appear after 2000 and polyps described as SSA/ Ps or TSAs were found only after 2010.

The individuals in our study were equally distributed in terms of sex (female: 47\%). However, the mean age of the cohort was 70 (range: 35-93) years, which is slightly 
higher than that found in previous studies [3, 26, 28, 30]. The age difference may, to some extent, be explained by the high proportion of dysplastic SPs in our study $(n=75$, $48 \%)$. Heredity, smoking and obesity have all been established as risk factors for SP, with smoking being more strongly linked to SSA/Ps than to the other subgroups [31-34]. In this study mention of risk factors in the patient chart was regarded as indicative of that risk factor, while, for instance, an individual in which smoking was not mentioned in the patient chart was regarded as a nonsmoker. Thus, the prevalence of some risk factors may have been underestimated. For instance, only $11 \%$ of our individuals had a record of obesity compared with $16 \%$ in the general Swedish population despite evidence showing that obesity is a risk factor for SP [31, 32, 35].

Several studies have established low detection as a significant challenge in SP research, and endoscopy screening seems less effective for detecting proximal CRC, which is believed to originate predominantly from the serrated pathway [6, 25, 36-39]. Moreover, HPs are considered less likely to bleed compared with adenomas, and SSA/Ps lack some genetic markers currently used in DNA faecal tests, decreasing the sensitivity of faecal tests for SPs.

In our study 15 (14\%) individuals had a positive FOBT prior to endoscopy and 58 (55\%) unique individuals had at least one sign of gastrointestinal bleeding (FOBT, haematochezia/melena or anaemia). To some extent the high percentage of individuals with SPs and signs of gastrointestinal bleeding can be explained by the simultaneous presence of adenomas $(n=18,31 \%)$, as well as the overrepresentation of SPs other than HPs. However, we cannot exclude that bleeding-prone SPs are overrepresented in our cohort.

Most individuals with SPs underwent endoscopy due to clinical symptoms $(n=64,60 \%)$. In addition, regardless of endoscopy indication, we found that 78 individuals $(74 \%)$ had at least one symptom (which includes positive FOBT) at the time of diagnosis, including one individual with a false positive SP. Of note, false positive cases more often presented with clinical symptoms as an indication for endoscopy ( $80 \%$ vs. $60 \%$ ).

In a notable proportion of HPs $(n=43,73 \%)$, grade of dysplasia was not specified. The reason for this is probably that HPs are normally defined as non-dysplastic. Thus, any specification of dysplasia by the pathologist would therefore be redundant considering that it is already implied by the HP diagnosis [40]. As such, the proportion of HPs without dysplasia should be interpreted as 97\% (59/61) instead of 26\% (16/61). Among the polyps classified as SAs, the vast majority exhibited low-grade dysplasia $(n=45,80 \%)$ and there were only three polyps (5\%) with no dysplasia. This observation reinforces the idea that polyps described as SAs are consistent with SSA/Ps, or possibly TSAs, as HPs are typically non-dysplastic [40]. More specifically, consistent with the literature on SSA/P location, we believe that proximal SAs will almost exclusively consist of SSA/Ps. However, SAs located in the rectum are likely to include a small number of TSAs.

The literature has shown that only about $15 \%$ of SSA/ Ps have any dysplastic features, implying that SSA/Ps with dysplasia are overrepresented in our study [28]. We cannot rule out that a few SSA/Ps with no dysplasia may have been misclassified as HPs given the established difficulty of distinguishing SSA/Ps from large proximal HPs [41]. Yet, it is also possible that SSA/Ps without dysplasia may have been overlooked and left undetected to a larger extent than SSA/Ps with dysplasia.

\section{Strengths and limitations}

The main strength of our study is the random selection of individuals with SPs from a nationwide histopathology cohort. Using a standardised form, we were able to examine not only the PPV for a histopathology report with SP but also describe Swedish individuals with SPs for clinical characteristics and risk factors. Our results are consistent with similar studies for which the gold standard of diagnosis is biopsy, further reinforcing the reliability of the present results.

A limitation of our study includes the lack of reexaminations of actual biopsies. The ethics review board allowed us to collect digital data but not actual tissue samples. Instead, the validation was based on re-evaluation of patient charts that included, among other things, histopathology and endoscopy reports. The quality of the patient chart data varied, especially in the documentation of risk factors and symptoms. Still, given that SP is a strictly histopathological diagnosis, the difference in data availability among the individuals should not have affected the validation in that all individuals had to have the corresponding histopathology report available to be included in the study.

Earlier studies have shown inter-observer variability for classification of SPs among pathologists [42, 43], and we cannot rule out some misclassification, especially for the subgroup classification. This could potentially affect the validity of SSA/P since some of the SSA/Ps may have been misdiagnosed as HPs, and vice versa [43]. The diversity of pathologists in this study, where some may not specialize in SPs, may have decreased the accuracy in polyp classification.

\section{Conclusion}

In conclusion, this study suggests that colorectal histopathology reports are a reliable data source to identify individuals with SPs. 


\section{Appendix}

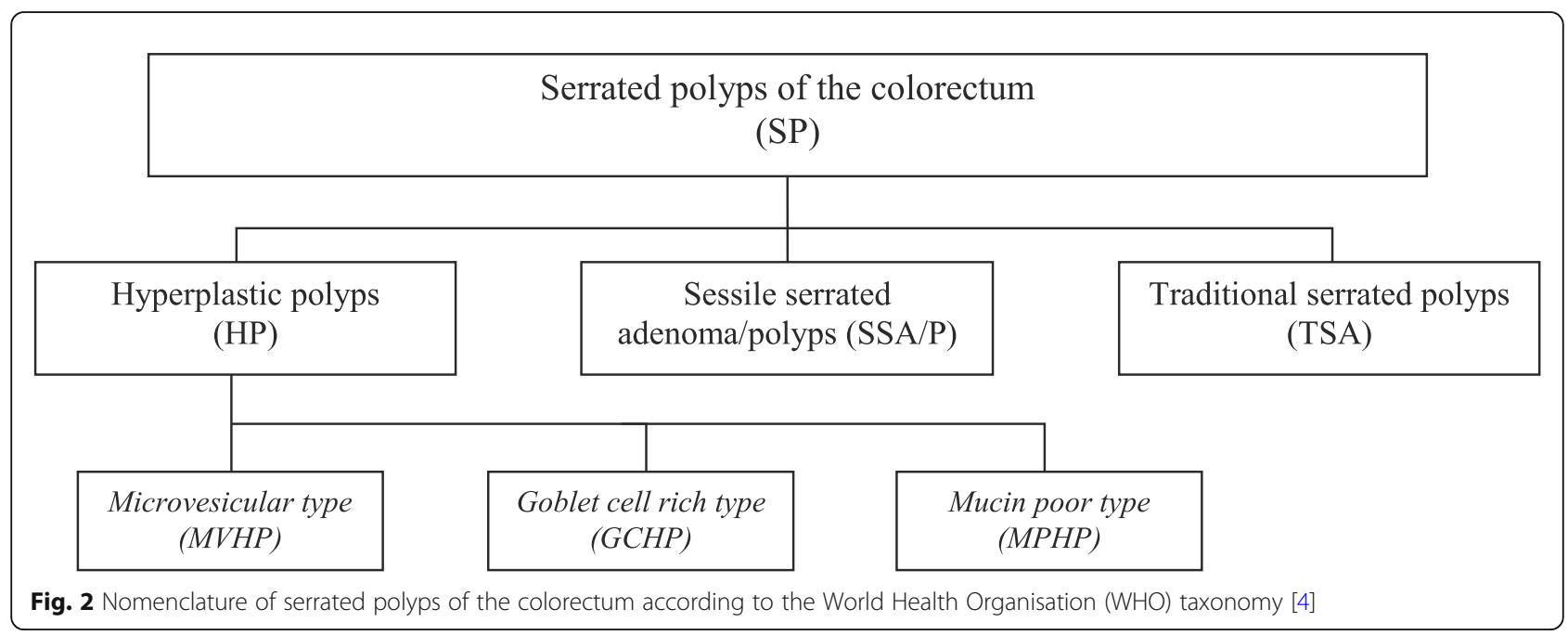

\section{Abbreviations}

Cl: Confidence interval; CRC: Colorectal cancer; FOBT: Faecal occult blood test; HP: Hyperplastic polyp; IBD: Inflammatory bowel disease; PPV: Positive predictive value; SNOMED: Systematised nomenclature of medicine; SP: Serrated polyp; SSA/P: Sessile serrated adenoma/polyp; TSA: Traditional serrated adenoma; WHO: World Health Organisation

\section{Acknowledgements}

Not applicable.

\section{Authors' contributions}

JFL was responsible for the conception and design of the study. SRB was responsible for the acquisition, analysis and interpretation of data, as well as drafting the manuscript. JFL, MS and LE contributed to the interpretation of data, and revision of the manuscript. All authors have approved of the manuscript before submission.

\section{Funding}

This research was supported by Karolinska Institutet and Union for International Cancer Control (Yamagiwa-Yoshida Award YY2/17/554363). The funders had no active role in the design of the study, the collection, analysis, and interpretation of data or in writing the manuscript.

\section{Availability of data and materials}

The datasets used and/or analysed during the current study are available from JFL on reasonable request.

\section{Ethics approval and consent to participate}

The study was approved by the Regional Ethical Review Board in Stockholm (July 19, 2017 with reference number: 2017/1497-32).

\section{Consent for publication}

Not applicable.

\section{Competing interests}

JFL coordinates a study on behalf of the Swedish IBD quality register (SWIBREG) which has received funding from the Janssen Corporation. The other authors declare no competing interests.

\section{Author details}

'Department of Medical Epidemiology and Biostatistics, Karolinska Institutet, 17177 Solna, Sweden. ²Departments of Epidemiology and Nutrition, Harvard T.H. Chan School of Public Health, Boston, MA, USA. ${ }^{3}$ Clinical and Translational Epidemiology Unit, Massachusetts General Hospital and Harvard Medical School, Boston, MA, USA. Division of Gastroenterology,
Massachusetts General Hospital and Harvard Medical School, Boston, MA, USA. Institute of Health and Society, University of Oslo, Oslo, Norway. 6Vårdcentralen Värmlands Nysäter and Centre for Clinical Research, County Council of Värmland, Värmland, Sweden. ${ }^{7}$ Department of Paediatrics, Örebro University Hospital, Örebro, Sweden. ${ }^{8}$ Division of Epidemiology and Public Health, School of Medicine, University of Nottingham, Nottingham, UK. ${ }^{9}$ Department of Medicine, Columbia University College of Physicians and Surgeons, New York, NY, USA.

Received: 5 May 2019 Accepted: 4 December 2019

Published online: 31 December 2019

\section{References}

1. Regionala cancercentrum i samverkan. Tjock- och ändtarmscancer: Nationellt vårdprogram. Regionala cancercentrum i samverkan. 2016 [cited 2018 Jan 29]. Available from https://www.cancercentrum.se/globalassets/ cancerdiagnoser/tjock\%2D\%2Doch-andtarm-anal/vardprogram/ nvpkolorektalcancer_2016-03-15.pdf.

2. Muto T, Bussey HJ, Morson BC. The evolution of cancer of the colon and rectum. Cancer. 1975:36(6):2251-70

3. Huang CS, Farraye FA, Yang S, O'Brien MJ. The clinical significance of serrated polyps. Am J Gastroenterol. 2011;106(2):229-40 quiz 41.

4. Bosman FT. WHO classification of tumours of the digestive system. Lyon: IARC Press; 2010.

5. Jass JR. Classification of colorectal cancer based on correlation of clinical, morphological and molecular features. Histopathology. 2007;50(1):113-30.

6. East JE, Saunders BP, Jass JR. Sporadic and syndromic hyperplastic polyps and serrated adenomas of the colon: classification, molecular genetics, natural history, and clinical management. Gastroenterol Clin N Am. 2008; 37(1):25-46 v.

7. Erichsen R, Baron JA, Hamilton-Dutoit SJ, Snover DC, Torlakovic EE, Pedersen $L$, et al. Increased risk of colorectal cancer development among patients with serrated polyps. Gastroenterology. 2016;150(4):895-902 e5.

8. Ludvigsson JF, Lashkariani M. Cohort profile: ESPRESSO (epidemiology strengthened by histoPathology reports in Sweden). Clin Epidemiol. 2019; 11:101-14.

9. Ludvigsson JF, Otterblad-Olausson P, Pettersson BU, Ekbom A. The Swedish personal identity number: possibilities and pitfalls in healthcare and medical research. Eur J Epidemiol. 2009;24(11):659-67.

10. Ludvigsson JF, Andersson E, Ekbom A, Feychting M, Kim JL, Reuterwall C, et al. External review and validation of the Swedish national inpatient register. BMC Public Health. 2011;11:450.

11. Brooke HL, Talback M, Hornblad J, Johansson LA, Ludvigsson JF, Druid $\mathrm{H}$, et al. The Swedish cause of death register. Eur J Epidemiol. 2017; 32(9):765-73. 
12. Barlow $L$, Westergren $K$, Holmberg $L$, Talback $M$. The completeness of the Swedish cancer register: a sample survey for year 1998. Acta Oncol. 2009; 48(1):27-33

13. Cnattingius S, Ericson A, Gunnarskog J, Kallen B. A quality study of a medical birth registry. Scand J Soc Med. 1990;18(2):143-8.

14. Wettermark B, Hammar N, Fored CM, Leimanis A, Otterblad Olausson P, Bergman $U$, et al. The new Swedish prescribed drug register-opportunities for pharmacoepidemiological research and experience from the first six months. Pharmacoepidemiol Drug Saf. 2007;16(7):726-35.

15. Ludvigsson JF, Svedberg P, Olen O, Bruze G, Neovius M. The longitudinal integrated database for health insurance and labour market studies (LISA) and its use in medical research. Eur J Epidemiol. 2019;34(4):423-37.

16. Ludvigsson JF, Almqvist C, Bonamy AK, Ljung R, Michaelsson K, Neovius M, et al. Registers of the Swedish total population and their use in medical research. Eur J Epidemiol. 2016:31(2):125-36.

17. Sergeant ESG. Epitools epidemiological calculators, Sample size to estimate a proportion or apparent prevalence with specified precision. Ausvet Pty Ltd.; 2019 [cited 2019 January 7]. Available from http://epitools.ausvet.com. au/content.php?page=1Proportion

18. Svensson M, Bergman D, Olen O, Myrelid P, Bohr J, Wickbom A, et al. Validating microscopic colitis (MC) in Swedish pathology registers. Scand J Gastroenterol. 2019:1-7.

19. Brown LD, Cai TT, DasGupta A. Interval estimation for a binomial proportion. Stat Sci. 2001;16(2):101-33.

20. Sergeant ESG. Epitools epidemiological calculators, Calculate confidence limits for a sample proportion. Ausvet Pty Ltd.; 2019 [cited 2019 January 7]. Available from http://epitools.ausvet.com.au/content.php?page= CIProportion.

21. Beutler E, Waalen J. The definition of anemia: what is the lower limit of normal of the blood hemoglobin concentration? Blood. 2006;107(5):1747.

22. East JE, Atkin WS, Bateman AC, Clark SK, Dolwani S, Ket SN, et al. British Society of Gastroenterology position statement on serrated polyps in the colon and rectum. Gut. 2017;66(7):1181-96.

23. Hamilton SR, Aaltonen LA. Pathology and genetics of tumours of the digestive system. Lyon: IARC press; 2000

24. Ludvigsson JF, Brandt L, Montgomery SM, Granath F, Ekbom A. Validation study of villous atrophy and small intestinal inflammation in Swedish biopsy registers. BMC Gastroenterol. 2009:9:19.

25. Hetzel JT, Huang CS, Coukos JA, Omstead K, Cerda SR, Yang S, et al. Variation in the detection of serrated polyps in an average risk colorectal cancer screening cohort. Am J Gastroenterol. 2010;105(12):2656-64.

26. Spring K, Zhao ZZ, Karamatic R, Walsh MD, Whitehall VL, Pike T, et al. High prevalence of sessile serrated adenomas with BRAF mutations: a prospective study of patients undergoing colonoscopy. Gastroenterology. 2006;131(5):1400-7.

27. Higuchi T, Sugihara K, Jass JR. Demographic and pathological characteristics of serrated polyps of colorectum. Histopathology. 2005;47(1):32-40.

28. Lash RH, Genta RM, Schuler CM. Sessile serrated adenomas: prevalence of dysplasia and carcinoma in 2139 patients. J Clin Pathol. 2010;63(8):681-6.

29. Carr NJ, Mahajan H, Tan KL, Hawkins NJ, Ward RL. Serrated and non-serrated polyps of the colorectum: their prevalence in an unselected case series and correlation of BRAF mutation analysis with the diagnosis of sessile serrated adenoma. J Clin Pathol. 2009;62(6):516-8.

30. Burnett-Hartman AN, Passarelli MN, Adams SV, Upton MP, Zhu LC, Potter JD, et al. Differences in epidemiologic risk factors for colorectal adenomas and serrated polyps by lesion severity and anatomical site. Am J Epidemiol. 2013;177(7):625-37.

31. Wallace K, Grau MV, Ahnen D, Snover DC, Robertson DJ, Mahnke D, et al. The association of lifestyle and dietary factors with the risk for serrated polyps of the colorectum. Cancer Epidemiol Biomark Prev. 2009;18(8):23107.

32. Bailie L, Loughrey MB, Coleman HG. Lifestyle risk factors for serrated colorectal polyps: a systematic review and meta-analysis. Gastroenterology. 2017;152(1):92-104.

33. Davenport JR, Su T, Zhao Z, Coleman HG, Smalley WE, Ness RM, et al. Modifiable lifestyle factors associated with risk of sessile serrated polyps, conventional adenomas and hyperplastic polyps. Gut. 2016.

34. Oines M, Helsingen LM, Bretthauer M, Emilsson L. Epidemiology and risk factors of colorectal polyps. Best Pract Res Clin Gastroenterol. 2017;31(4): 419-24

35. Folkhälsomyndigheten. Folkhälsodata. Solna: Folkhälsomyndigheten; 2018 [cited 2018 January 7]. Available from http://fohm-app.
folkhalsomyndigheten.se/Folkhalsodata/pxweb/sv/B_HLV/B_HLV_ aLevvanor aahLevvanorvikt/HLV Viktobmi_alder.px/table/ tableViewLayout1/?rxid=b74d8053-7b13-4cfa-8716-aa2a5c66f44c.

36. Crockett SD, Snover DC, Ahnen DJ, Baron JA. Sessile serrated adenomas: an evidence-based guide to management. Clin Gastroenterol Hepatol. 2015; 13(1):11-26 e1.

37. Kahi CJ, Hewett DG, Norton DL, Eckert GJ, Rex DK. Prevalence and variable detection of proximal colon serrated polyps during screening colonoscopy. Clin Gastroenterol Hepatol. 2011;9(1):42-6.

38. Baxter NN, Goldwasser MA, Paszat LF, Saskin R, Urbach DR, Rabeneck L. Association of colonoscopy and death from colorectal cancer. Ann Intern Med. 2009;150(1):1-8.

39. Nishihara R, Wu K, Lochhead P, Morikawa T, Liao X, Qian ZR, et al. Longterm colorectal-cancer incidence and mortality after lower endoscopy. $\mathrm{N}$ Engl J Med. 2013:369(12):1095-105

40. Torlakovic EE, Gomez JD, Driman DK, Parfitt JR, Wang C, Benerjee T, et al. Sessile serrated adenoma (SSA) vs. traditional serrated adenoma (TSA). Am J Surg Pathol. 2008;32(1):21-9.

41. O'Connell BM, Crockett SD. The clinical impact of serrated colorectal polyps. Clin Epidemiol. 2017;9:113-25.

42. Leggett $B$, Whitehall $V$. Role of the serrated pathway in colorectal cancer pathogenesis. Gastroenterology. 2010;138(6):2088-100.

43. Farris AB, Misdraji J, Srivastava A, Muzikansky A, Deshpande V, Lauwers GY, et al. Sessile serrated adenoma: challenging discrimination from other serrated colonic polyps. Am J Surg Pathol. 2008;32(1):30-5.

\section{Publisher's Note}

Springer Nature remains neutral with regard to jurisdictional claims in published maps and institutional affiliations.
Ready to submit your research? Choose BMC and benefit from:

- fast, convenient online submission

- thorough peer review by experienced researchers in your field

- rapid publication on acceptance

- support for research data, including large and complex data types

- gold Open Access which fosters wider collaboration and increased citations

- maximum visibility for your research: over $100 \mathrm{M}$ website views per year

At $\mathrm{BMC}$, research is always in progress.

Learn more biomedcentral.com/submissions 